\title{
International Journal of Pharmacognosy and Chemistry
}

Open Access

Research Article

Formulation development and evaluation of orally disintegrating tablets rizatriptan benzoate

\section{Ch,Saibabu ${ }^{1 *}$, P.Triveni ${ }^{2}$}

${ }^{1}$ Head , Department of Pharmaceutics, M.L. College of Pharmacy, S. Konda-523101

${ }^{2}$ Department of Pharmaceutics, M.L. College of Pharmacy, S. Konda-523101

\begin{tabular}{l}
\hline Article History \\
\hline Received on: 15-08-2021 \\
Revised on : 29-08-2021 \\
Accepted on : 12-10-2021 \\
\hline $\begin{array}{l}\text { Keywords: Orally disintegrating } \\
\text { tablets, Superdisintegrants, Rizatrip- } \\
\text { tan benzoate, Direct compression. }\end{array}$
\end{tabular}

\section{DOI:}

https://doi.org/10.46796/ijpc.v2i4.246

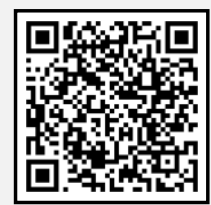

\section{Abstract}

Formulation research is oriented towards safety, efficacy and quick onset of action of existing drug molecule through novel concepts of drug delivery. Orally disintegrating tablets of Rizatriptan benzoate were prepared by direct compression method to provide faster relief from pain to migraine sufferers. About eleven formulations for the present study were carried out. Croscarmellose sodium, Crospovidone and Sodium starch glycolate (SSG) were used as superdisintegrants, while microcrystalline cellulose was used as diluent. The prepared batches of tablets were evaluated for weight variation, hardness, friability, wetting time, invitro dispersion time, drug content and invitro dissolution studies. The formulation containing combination of Croscarmellose sodium and Sodium starch glycolate showed rapid invitro dispersion time as compared to other formulations. The optimized formulation dispersed in 8 seconds. It also showed a higher water absorption ratio and $99.58 \%$ of drug is released within 2 minutes.

This article is licensed under a Creative Commons Attribution-Non Commercial 4.0 International License. Copyright (c) 2021 Author(s) retain the copyright of this article.

\section{*Corresponding Author}

Ch,Saibabu

Email ID: saichennupalli@gmail.com

\section{Introduction}

Oral Disintegrating Tablets (ODTs) [1,2] are solid dosage forms containing medicinal substances which disintegrate rapidly, usually in a matter of seconds, when placed on the tongue. The marketed drug of Rizatriptan is available under the brand name Maxalt-MLT25 in 5 $\mathrm{mg}$ and $10 \mathrm{mg}$ strengths. Maxalt-MLT is prepared using freeze drying technique which is very cumbersome process and costly. Further the product obtained is very brittle which requires special attention during packing and removal from the pack. So, the aim of the present study is to develop better formulation of Rizatriptan that is having good stability using a cost effective process.

Rizatriptan Benzoate is an Anti migraine drug that is white to off white crystalline solid. Migraine $[3,4]$ is a specific and common form of headache that has been known since antiquity. It is traditionally called as vascular headache due to the belief that it is due to abnormal changes in the blood vessel tone. The symptoms of a migraine may include throbbing or dull aching pain on one or both sides of the head nausea, vomiting, diarrhea, blurred vision or blind spots, anxious or restlessness, light headedness, tender scalp, cold hands and feet. The therapeutic activity of Rizatriptan in migraine can most likely be attributed to agonist effects at 5-HT1B/1D receptors on the extra 
cerebral, intracranial blood vessels that become dilated during a migraine attack and on nerve terminals in the trigeminal system thus causing vaso constriction and inhibition of neuropeptide release and reduced transmission in trigeminal pain pathways and thus subsequent relief of migraine headache is seen.

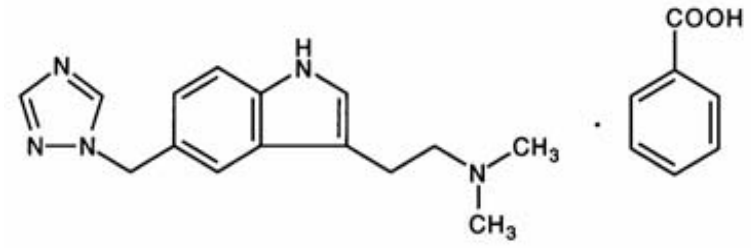

Figure 01: chemical structure of Rizatriptan Benzoate

\section{Experimental work [5-6]}

\section{Materials and methods}

Rizatriptan Benzoate USP, as a gift sample from Matrix laboratories Ltd, hyd. Lactose Spray Dried, Avicel ph 102 , Pearlitol SD 200 , Crosspovidone XL ,Cross carmellose sodiumSodium Starch Glycolate , Aspartame , Mint cream flavor , Magnesium stearate are purchased from Signet chemical corporation Mumbai. All other chemicals used were of analytical grade.

\section{Methods}

Preformulation study can divide in to two Subclasses.
1. API characterization,
2. Compatibility study

\section{Manufacturing Process}

Formulation of oral disintegrating tablets of Rizatriptan $5 \mathrm{mg}$ and 10mg were carried out by direct compression technique.

\section{Formulation Development For 5mg Tablets}

Table 01: Formulation development for $5 \mathbf{~ m g}$ tablets

\begin{tabular}{|c|c|c|c|c|c|c|c|c|c|c|c|c|}
\hline S.NO & Ingredients & F1 & F2 & F3 & F4 & F5 & F6 & F7 & F8 & F9 & F10 & F11 \\
\hline 1 & $\begin{array}{c}\text { Rizatriptan Ben- } \\
\text { zoate }\end{array}$ & 7.26 & 7.26 & 7.26 & 7.26 & 7.26 & 7.26 & 7.26 & 7.26 & 7.26 & 7.26 & 7.26 \\
\hline 2 & $\begin{array}{c}\text { Spray dried Lac- } \\
\text { tose }\end{array}$ & 25.19 & - & - & - & - & - & - & - & - & - & - \\
\hline 3 & Avicel pH102 & - & 41.99 & 58.79 & 25.19 & 25.19 & 25.19 & 25.49 & 24.89 & 25.38 & 25.01 & 33.59 \\
\hline 4 & Pearlitol 200 SD & 58.79 & 41.99 & 5.19 & 58.79 & 58.79 & 58.79 & 59.49 & 58.09 & 59.23 & 58.35 & 50.38 \\
\hline 5 & \begin{tabular}{c} 
Cross povidone \\
\hline 6
\end{tabular} & 5 & 5 & 5 & 5 & - & - & 4 & 6 & 5 & 5 & 5 \\
\hline 7 & $\begin{array}{c}\text { Cross carmellose } \\
\text { sodium }\end{array}$ & - & - & - & - & 5 & - & - & - & - & - & - \\
\hline 8 & Sodium starch & - & - & - & - & - & 5 & - & - & - & - & - \\
\hline 9 & $\begin{array}{c}\text { Peppermint fla- } \\
\text { vour }\end{array}$ & 0.5 & 0.5 & 0.5 & 0.5 & 0.5 & 0.5 & 0.5 & 0.5 & 0.5 & 0.5 & 0.5 \\
\hline 10 & $\begin{array}{c}\text { Magnesium } \\
\text { stearate }\end{array}$ & 1.25 & 1.25 & 1.25 & 1.25 & 1.25 & 1.25 & 1.25 & 1.25 & 0.62 & 1.87 & 1.25 \\
\hline & \begin{tabular}{c} 
Total weight(mg) \\
\hline
\end{tabular} & 100 & 100 & 100 & 100 & 100 & 100 & 100 & 100 & 100 & 100 & 100 \\
\hline
\end{tabular}


Formulation Development For $10 \mathrm{mg}$ Tablets

Table 02: Formulation development for $10 \mathrm{mg}$ tablets

\begin{tabular}{|c|c|c|c|c|c|c|c|c|c|c|c|c|}
\hline S.NO & Ingredients & F1 & F2 & F3 & F4 & F5 & F6 & F7 & F8 & F9 & F10 & $\begin{array}{c}\text { F1 } \\
1\end{array}$ \\
\hline 1 & $\begin{array}{c}\text { Rizatriptan } \\
\text { Benzoate }\end{array}$ & 14.53 & 14.53 & 14.53 & 14.53 & 14.53 & 14.53 & 14.53 & 14.53 & 14.53 & 14.53 & $\begin{array}{c}14 \\
.5 \\
3\end{array}$ \\
\hline 2 & $\begin{array}{l}\text { Spray dried } \\
\text { Lactose }\end{array}$ & 50.39 & - & - & - & - & - & - & - & - & - & - \\
\hline 3 & Avicel pH102 & - & 83.98 & 117.57 & 50.39 & 50.39 & 50.39 & 50.99 & 49.79 & 50.76 & 50.01 & $\begin{array}{c}50 \\
.3 \\
9\end{array}$ \\
\hline 4 & Pearlitol 200SD & 117.57 & 83.98 & 50.39 & 117.57 & 117.57 & 117.57 & $\begin{array}{c}118.9 \\
7\end{array}$ & $\begin{array}{c}116.1 \\
7\end{array}$ & $\begin{array}{c}118.4 \\
5\end{array}$ & $\begin{array}{c}116.7 \\
0\end{array}$ & $\begin{array}{l}11 \\
7 . \\
57\end{array}$ \\
\hline 5 & Cross povidone & 10 & 10 & 10 & 10 & - & - & 8 & 12 & 10 & 10 & 10 \\
\hline 6 & $\begin{array}{l}\text { Cross carmel- } \\
\text { lose sodium }\end{array}$ & - & - & - & - & 10 & - & - & - & - & - & - \\
\hline 7 & $\begin{array}{l}\text { Sodium starch } \\
\text { glycollate }\end{array}$ & - & - & - & - & - & 10 & - & - & - & - & - \\
\hline 8 & Aspartame & 4 & 4 & 4 & 4 & 4 & 4 & 4 & 4 & 4 & 4 & 4 \\
\hline 9 & $\begin{array}{l}\text { Peppermint } \\
\text { flavor }\end{array}$ & 1 & 1 & 1 & 1 & 1 & 1 & 1 & 1 & 1 & 1 & 1 \\
\hline 10 & $\begin{array}{l}\text { Magnesium } \\
\text { stearate }\end{array}$ & 2.5 & 2.5 & 2.5 & 2.5 & 2.5 & 2.5 & 2.5 & 2.5 & 1.25 & 3.75 & $\begin{array}{l}2 . \\
5\end{array}$ \\
\hline & $\begin{array}{c}\text { Total } \\
\text { weight(mg) }\end{array}$ & 200 & 200 & 200 & 200 & 200 & 200 & 200 & 200 & 200 & 200 & $\begin{array}{c}20 \\
0\end{array}$ \\
\hline
\end{tabular}

1. API Characterization

Organoleptic evaluation

Loss on drying

Particle size analysis:

Angle of repose

Bulk density

Tapped density

Compressibility index

Hausner ratio

Solubility studies

Characterisation Of Rizatriptan Benzoate USP [ 7]

Characterisation Of Rizatriptan Benzoate Usp [7]

Table 03: Characterization of Rizatriptan benzoate

\begin{tabular}{|c|c|c|}
\hline Test & Result & Specification \\
\hline Description & Crystalline white & Crystalline \\
\hline
\end{tabular}

\begin{tabular}{|c|c|c|}
\hline Solubility & $\begin{array}{c}\text { powder. } \\
\text { test }\end{array}$ & $\begin{array}{c}\text { white to off } \\
\text { white powder. }\end{array}$ \\
\hline $\begin{array}{c}\text { Bulk density } \\
\text { (g/ml) }\end{array}$ & 0.43 & $\begin{array}{c}\text { to be soluble } \\
\text { in water and } \\
\text { methanol }\end{array}$ \\
\hline $\begin{array}{c}\text { tapped den- } \\
\text { sity (g/ml) }\end{array}$ & 0.82 & $0.38-0.69$ \\
\hline $\begin{array}{c}\text { Loss on dry- } \\
\text { ing (\%) }\end{array}$ & 0.12 & NMT 0.5 \\
\hline Assay (\%) & 99.8 & $\begin{array}{c}\text { NLT 99.0\& } \\
\text { NMT 101.0 }\end{array}$ \\
\hline $\begin{array}{c}\text { Residue on } \\
\text { ignition }\end{array}$ & 0.03 & NMT 0.1 \\
\hline
\end{tabular}




\begin{tabular}{|c|c|c|}
\hline $\mathbf{( \% w} / \mathbf{w})$ & & \\
\hline $\begin{array}{c}\text { Benzoic acid } \\
\text { (chemical)(\%) }\end{array}$ & 31.3 & $\begin{array}{c}\text { NLT } \\
30.8 \& N M T \\
31.5\end{array}$ \\
\hline $\begin{array}{c}\text { Particle } \\
\text { size(microns) }\end{array}$ & 30.4 & NLT 50 \\
\hline $\begin{array}{c}\text { Melting } \\
\left.\text { point( }{ }^{\circ} \mathbf{C}\right)\end{array}$ & 178 & $\begin{array}{c}\text { NLT } \\
\text { 178\&NMT 180 }\end{array}$ \\
\hline
\end{tabular}

\section{Solublity Studies}

Table 04: Solubility studies of Rizatriptan benzoate

\begin{tabular}{|c|c|c|c|}
\hline S.No. & Media & $\begin{array}{c}\text { Solublity } \\
\text { For 5mg } \\
\text { Tablets } \\
\text { (Mg/Ml) }\end{array}$ & $\begin{array}{c}\text { Solublity } \\
\text { For 10mg } \\
\text { Tablets } \\
\text { (Mg/Ml) }\end{array}$ \\
\hline 1 & purified water & 0.03 & 0.06 \\
\hline 2 & pH1.2 buffer & 0.03 & 0.06 \\
\hline 3 & $\begin{array}{c}\text { pH4.5 acetate } \\
\text { buffer }\end{array}$ & 0.03 & 0.06 \\
\hline 4 & $\begin{array}{c}\text { pH6.8 phos- } \\
\text { phate buffer }\end{array}$ & 0.03 & 0.06 \\
\hline
\end{tabular}

Results of Precompression Parameters

Table 05: Precompression results of blend

\begin{tabular}{|c|c|c|c|c|c|c|}
\hline $\begin{array}{c}\text { S.N } \\
\text { O }\end{array}$ & $\begin{array}{c}\text { Formu- } \\
\text { lation } \\
\text { Code }\end{array}$ & $\begin{array}{c}\text { Bulk } \\
\text { den- } \\
\text { sity } \\
(\mathrm{g} / \mathrm{ml})\end{array}$ & $\begin{array}{c}\text { Tap } \\
\text { ped } \\
\text { den- } \\
\text { sity } \\
(\mathrm{g} / \mathrm{m} \\
1)\end{array}$ & $\begin{array}{c}\text { Car } \\
\text { r's } \\
\text { in- } \\
\text { dex }\end{array}$ & $\begin{array}{l}\text { Haus } \\
\text { ners } \\
\text { ratio }\end{array}$ & $\begin{array}{l}\text { An } \\
\text { gle } \\
\text { of } \\
\text { re- } \\
\text { po } \\
\text { se } \\
(\theta)\end{array}$ \\
\hline 1 & F1 & 0.58 & 0.79 & $\begin{array}{c}26.5 \\
8\end{array}$ & 1.36 & 25 \\
\hline 2 & F2 & 0.68 & 0.76 & 13.1 & 1.11 & 26 \\
\hline 3 & F3 & 0.67 & 0.78 & 12.8 & 1.16 & 28 \\
\hline 4 & $\mathrm{~F} 4$ & 0.59 & 0.65 & 9.2 & 1.10 & 24 \\
\hline 5 & F5 & 0.62 & 0.70 & 11.4 & 1.12 & 27 \\
\hline 6 & F6 & 0.59 & 0.66 & 10.6 & 1.11 & 25 \\
\hline 7 & F7 & 0.63 & 0.71 & 11.2 & 1.12 & 27 \\
\hline 8 & F8 & 0.66 & 0.73 & 9.5 & 1.10 & 24 \\
\hline 9 & F9 & 0.66 & 0.74 & 10.8 & 1.12 & 32 \\
\hline 10 & F10 & 0.58 & 0.64 & 9.37 & 1.10 & 23 \\
\hline 11 & F11 & 0.60 & 0.66 & 9.0 & 1.10 & 23 \\
\hline
\end{tabular}

Drug Excipient Compatiblity Studies Of Rizatriptan ODT's

Table 06: Drug Excipient compatibility studies

\begin{tabular}{|c|c|c|c|}
\hline \multirow[b]{2}{*}{ Ingredient } & \multirow[b]{2}{*}{$\begin{array}{l}\text { Ra- } \\
\text { tio }\end{array}$} & \multicolumn{2}{|c|}{ Description } \\
\hline & & INITIAL & $\begin{array}{c}\text { FI- } \\
\text { NAL } \\
40^{0} \pm \\
2^{\circ} \mathrm{C} / \\
75 \% \pm 5 \\
\% \\
\text { RH( } 4 \\
\text { weeks) }\end{array}$ \\
\hline Rizatriptan benzoate & 1 & $\begin{array}{l}\text { white } \\
\text { powder }\end{array}$ & $\begin{array}{c}\text { No } \\
\text { chang } \\
\mathrm{e}\end{array}$ \\
\hline Spray dried Lactose & 1 & $\begin{array}{l}\text { White } \\
\text { powder }\end{array}$ & $\begin{array}{c}\text { No } \\
\text { chang } \\
\mathrm{e}\end{array}$ \\
\hline Avicel Ph 102 & 1 & $\begin{array}{c}\text { white } \\
\text { crystal- } \\
\text { line pow- } \\
\text { der }\end{array}$ & $\begin{array}{c}\text { No } \\
\text { chang } \\
\mathrm{e}\end{array}$ \\
\hline Pearlitol 200 SD & 1 & $\begin{array}{c}\text { white } \\
\text { crystal- } \\
\text { line pow- } \\
\text { der }\end{array}$ & $\begin{array}{c}\text { No } \\
\text { chang } \\
\text { e }\end{array}$ \\
\hline Cross povidone & 1 & $\begin{array}{c}\text { white } \\
\text { crystal- } \\
\text { line pow- } \\
\text { der }\end{array}$ & $\begin{array}{c}\text { No } \\
\text { chang } \\
\text { e }\end{array}$ \\
\hline $\begin{array}{l}\text { Cross carmellose } \\
\text { sodium }\end{array}$ & 1 & $\begin{array}{l}\text { white } \\
\text { powder }\end{array}$ & $\begin{array}{c}\text { No } \\
\text { chang } \\
\mathrm{e}\end{array}$ \\
\hline $\begin{array}{l}\text { Sodium starch gly- } \\
\text { collate }\end{array}$ & 1 & $\begin{array}{l}\text { White } \\
\text { powder }\end{array}$ & $\begin{array}{c}\text { No } \\
\text { chang } \\
\mathrm{e}\end{array}$ \\
\hline Aspartame & 1 & $\begin{array}{l}\text { white } \\
\text { powder }\end{array}$ & $\begin{array}{c}\text { No } \\
\text { chang } \\
\mathrm{e}\end{array}$ \\
\hline Peppermint flavor & 1 & $\begin{array}{l}\text { white } \\
\text { powder }\end{array}$ & $\begin{array}{c}\text { No } \\
\text { chang } \\
\mathrm{e}\end{array}$ \\
\hline Magnesium stearate & 1 & $\begin{array}{c}\text { white } \\
\text { powder }\end{array}$ & $\begin{array}{c}\text { No } \\
\text { chang } \\
\mathrm{e}\end{array}$ \\
\hline $\begin{array}{l}\text { Rizatriptan benzo- } \\
\text { ate+spray dried lac- } \\
\text { tose }\end{array}$ & $1: 20$ & $\begin{array}{l}\text { white } \\
\text { powder }\end{array}$ & $\begin{array}{c}\text { No } \\
\text { chang } \\
\text { e }\end{array}$ \\
\hline
\end{tabular}




\begin{tabular}{|c|c|c|c|}
\hline $\begin{array}{l}\text { Rizatriptan benzo- } \\
\text { ate+avicel pH102 }\end{array}$ & $1: 20$ & $\begin{array}{c}\text { White } \\
\text { crystal- } \\
\text { line pow- } \\
\text { der }\end{array}$ & $\begin{array}{c}\text { No } \\
\text { chang } \\
\text { e }\end{array}$ \\
\hline $\begin{array}{l}\text { Rizatriptan benzo- } \\
\text { ate+ Pearlitol } 200 \text { SD }\end{array}$ & $1: 20$ & $\begin{array}{c}\text { White } \\
\text { crystal- } \\
\text { line pow- } \\
\text { der }\end{array}$ & $\begin{array}{c}\text { No } \\
\text { chang } \\
\text { e }\end{array}$ \\
\hline $\begin{array}{l}\text { Rizatriptan benzo- } \\
\text { ate+cross povidone }\end{array}$ & $1: 2$ & $\begin{array}{l}\text { White } \\
\text { cryst } \\
\text { powder }\end{array}$ & $\begin{array}{c}\text { No } \\
\text { chang } \\
\text { e }\end{array}$ \\
\hline $\begin{array}{l}\text { Rizatriptan benzo- } \\
\text { ate+cross carmellose } \\
\text { sodium }\end{array}$ & $1: 2$ & $\begin{array}{l}\text { White } \\
\text { powder }\end{array}$ & $\begin{array}{c}\text { No } \\
\text { chang } \\
\text { e }\end{array}$ \\
\hline $\begin{array}{l}\text { Rizatriptan benzo- } \\
\text { ate+sodium starch } \\
\text { glycollate }\end{array}$ & $1: 2$ & $\begin{array}{l}\text { White } \\
\text { powder }\end{array}$ & $\begin{array}{c}\text { No } \\
\text { chang } \\
\text { e }\end{array}$ \\
\hline $\begin{array}{l}\text { Rizatriptan benzo- } \\
\text { ate+aspartame }\end{array}$ & 1:0.5 & $\begin{array}{l}\text { white } \\
\text { powder }\end{array}$ & $\begin{array}{c}\text { No } \\
\text { chang } \\
\text { e }\end{array}$ \\
\hline $\begin{array}{l}\text { Rizatriptan benzo- } \\
\text { ate+peppermint fla- } \\
\text { vor }\end{array}$ & 1:0.5 & $\begin{array}{l}\text { White } \\
\text { powder }\end{array}$ & $\begin{array}{c}\text { No } \\
\text { chang } \\
\text { e }\end{array}$ \\
\hline $\begin{array}{l}\text { Rizatriptan benzo- } \\
\text { ate+ magnesium } \\
\text { stearate }\end{array}$ & 1:0.5 & $\begin{array}{l}\text { White } \\
\text { powder }\end{array}$ & $\begin{array}{c}\text { No } \\
\text { chang } \\
\text { e }\end{array}$ \\
\hline
\end{tabular}

Evaluation Parameters of Orally Disintegrating Tablets

\section{Weight variation test [8]}

Weight variation test was done by weighing 20 tablets individually, by using analytical balance. Calculating the average weight and comparing the individual tablet weight to the average weight.

\section{Tablet thickness [8]}

The thickness was measured by placing tablet between two arms of the Vernier calipers. 5 tablets were taken and their thickness was measured.

Tablet hardness [8]

The tablet hardness, which is the force required to break a tablet in a diametric compression force. The hardness tester used in the study was Monsanto hardness tester, which applies force to the tablet diametrically with the help of an inbuilt spring.

Tablet friability [8]

The friability of the tablets was measured in a Roche friabilator. Tablets of a known weight (W0) are dedusted in a drum for a fixed time (100 revolutions) and weighed
(W) again. Percentage friability was calculated from the loss in weight as given in equation as below. The weight loss should not be more than $1 \%$. Determination was made in triplicate.

$$
\% \text { Friability }=\frac{(\text { Initial weight }- \text { Final weight })}{\text { Initial weight }} \times 100
$$

\section{Wetting time [9]}

The wetting time of the tablets can be measured using a simple procedure. Five circular tissue papers of $10 \mathrm{~cm}$ diameter are placed in a petridish with a $10 \mathrm{~cm}$ diameter. Ten millimeters of water- containing Eosin, a water soluble dye, is added to petridish. A tablet is carefully placed on the surface of the tissuepaper. The time required for water to reach upper surface of the tablet is noted as a wetting time.

\section{Water absorption ratio (\%) [10-11]}

A piece of tissue paper folded twice was placed in a small petridish $($ Internal Diameter $=6.5 \mathrm{~cm}$ ) containing 6 $\mathrm{ml}$ of water. A tablet was placed on the paper and the time required for complete wetting was then measured. The water absorption ratio $(\mathrm{R})$ was determined using the following equation.

$$
R=\frac{W a-W b}{W b}
$$

Where, $\mathrm{Wb}$ is the weight of the tablet before water absorption and $\mathrm{Wa}$ is the weight of the tablet after water absorption.

\section{In-vitro dissolution study [12-14]}

The release rate of Rizatriptan benzoate from orally disintegrating tablets was determined using United State Pharmacopoeia (USP) XXIV dissolution testing apparatus II (paddle method). The dissolution test was performed using $900 \mathrm{ml}$ of $0.1 \mathrm{~N} \mathrm{HCl} \mathrm{pH} 1.2$ as a dissolution medium, at $37 \pm 0.5^{\circ} \mathrm{C}$ and $50 \mathrm{rpm}$. A sample $(5 \mathrm{ml})$ of the solution was withdrawn from the dissolution apparatus at 1, 2, 3, 4, 5, 10, 20 and $30 \mathrm{~min}$. The samples were filtered through a 0.45 membrane filter. Absorbance of these solutions was measured at $280 \mathrm{~nm}$ using a Shimadzu UV-1700 UV/VIS spectrophotometer. Cumulative percentage of drug release was calculated using an equation obtained from a standard curve.

\section{Post Compression Parameters Of 5 Mg Tablets}

\begin{tabular}{|c|c|c|c|c|c|c|}
\hline $\begin{array}{l}\text { For- } \\
\text { mula- } \\
\text { tion } \\
\text { Code }\end{array}$ & $\begin{array}{c}\text { Av } \\
\text { er- } \\
\text { age } \\
\text { wei } \\
\text { ght } \\
\text { (mg }\end{array}$ & $\begin{array}{c}\text { Thic } \\
\text { knes } \\
\text { s } \\
(\mathrm{mm} \\
\text { ) }\end{array}$ & $\begin{array}{c}\text { Har } \\
\text { dne } \\
\text { ss } \\
\text { (kp) }\end{array}$ & $\begin{array}{l}\text { Per- } \\
\text { cent- } \\
\text { age } \\
\text { Fri- } \\
\text { abil- } \\
\text { ity }\end{array}$ & $\begin{array}{l}\text { Disin- } \\
\text { tegra- } \\
\text { tion } \\
\text { Time } \\
\text { (sec) }\end{array}$ & $\begin{array}{l}\text { Wet } \\
\text { ting } \\
\text { tim } \\
\text { e(se } \\
\text { c) }\end{array}$ \\
\hline
\end{tabular}

Table 0 7: Physical parameters of $5 \mathrm{mg}$ tablets 


\begin{tabular}{|c|c|c|c|c|c|c|}
\hline F1 & $\begin{array}{c}100 . \\
1\end{array}$ & 2.78 & 1.09 & 1.32 & 18 & 12 \\
\hline F2 & $\begin{array}{c}100 . \\
2\end{array}$ & 3.12 & 2.01 & 0.19 & 19 & 11 \\
\hline F3 & $\begin{array}{c}100 . \\
0\end{array}$ & 2.97 & 1.75 & 0.35 & 10 & 8 \\
\hline F4 & $\begin{array}{c}100 . \\
0\end{array}$ & 2.83 & 1.73 & 0.38 & 9 & 6 \\
\hline F5 & 99.8 & 3.01 & 1.75 & 0.37 & 14 & 10 \\
\hline F6 & $\begin{array}{c}100 . \\
1\end{array}$ & 2.88 & 1.76 & 0.36 & 18 & 11 \\
\hline F7 & $\begin{array}{c}100 . \\
3\end{array}$ & 2.68 & 1.73 & 0.37 & 12 & 8 \\
\hline F8 & $\begin{array}{c}100 . \\
0\end{array}$ & 2.79 & 1.73 & 0.38 & 9 & 6 \\
\hline F9 & 99.7 & 2.75 & 1.71 & 0.42 & 10 & 7 \\
\hline F10 & 99.9 & 2.81 & 1.76 & 0.34 & 11 & 7 \\
\hline F11 & 99.9 & 2.81 & 1.74 & 0.38 & 9 & 6 \\
\hline
\end{tabular}

\begin{tabular}{|l|c|c|c|c|c|c|}
\hline F11 & $\begin{array}{c}200 . \\
00\end{array}$ & 2.95 & 1.74 & 0.34 & 11 & 7 \\
\hline
\end{tabular}

\section{Dissolution Studies For Rizatriptan ODT}

Cumulative \% drug release of $5 \mathrm{mg}$ tablets

Table 09: Cumulative \% drug release of $5 \mathrm{mg}$ tablets

\begin{tabular}{|c|c|c|c|c|c|c|c|c|c|c|c|c|}
\hline $\begin{array}{l}\text { S. } \\
\mathbf{N} \\
\text { O }\end{array}$ & $\begin{array}{c}\text { TI } \\
\text { ME } \\
\text { (mi } \\
\text { n) }\end{array}$ & $\begin{array}{l}\mathbf{F} \\
\mathbf{1}\end{array}$ & $\begin{array}{l}F \\
2\end{array}$ & $\begin{array}{l}F \\
3\end{array}$ & $\begin{array}{l}F \\
4\end{array}$ & $\begin{array}{l}F \\
5\end{array}$ & $\begin{array}{l}F \\
6\end{array}$ & $\begin{array}{l}\text { F } \\
7\end{array}$ & $\begin{array}{l}F \\
8\end{array}$ & $\begin{array}{l}F \\
9\end{array}$ & $\begin{array}{l}F \\
1 \\
0\end{array}$ & $\begin{array}{l}F \\
1 \\
1\end{array}$ \\
\hline 1 & 0 & 0 & 0 & 0 & 0 & 0 & 0 & 0 & 0 & 0 & 0 & 0 \\
\hline 2 & 5 & $\begin{array}{l}8 \\
8 \\
. \\
9\end{array}$ & $\begin{array}{l}8 \\
9 \\
. \\
3\end{array}$ & $\begin{array}{l}9 \\
0 \\
. \\
0\end{array}$ & $\begin{array}{l}8 \\
9 . \\
8\end{array}$ & $\begin{array}{l}8 \\
9 \\
. \\
2\end{array}$ & $\begin{array}{l}8 \\
8 \\
. \\
9\end{array}$ & $\begin{array}{l}8 \\
9 \\
. \\
5\end{array}$ & $\begin{array}{l}8 \\
9 . \\
9\end{array}$ & $\begin{array}{l}8 \\
9 \\
. \\
5\end{array}$ & $\begin{array}{l}8 \\
9 . \\
8\end{array}$ & $\begin{array}{l}9 \\
0 \\
. \\
0\end{array}$ \\
\hline 3 & 10 & $\begin{array}{l}9 \\
5 \\
. \\
2\end{array}$ & $\begin{array}{l}9 \\
5 \\
. \\
9\end{array}$ & $\begin{array}{l}9 \\
6 \\
. \\
2\end{array}$ & $\begin{array}{c}9 \\
7 . \\
1\end{array}$ & $\begin{array}{l}9 \\
6 \\
. \\
8\end{array}$ & $\begin{array}{l}9 \\
6 \\
. \\
3\end{array}$ & $\begin{array}{l}9 \\
6 \\
. \\
8\end{array}$ & $\begin{array}{l}9 \\
7 . \\
3\end{array}$ & $\begin{array}{l}9 \\
7 \\
. \\
1\end{array}$ & $\begin{array}{l}9 \\
7 . \\
2\end{array}$ & $\begin{array}{l}9 \\
7 \\
. \\
5\end{array}$ \\
\hline 4 & 15 & $\begin{array}{l}9 \\
8 \\
. \\
8\end{array}$ & $\begin{array}{l}9 \\
9 \\
. \\
1\end{array}$ & $\begin{array}{l}9 \\
9 \\
. \\
6\end{array}$ & $\begin{array}{l}1 \\
0 \\
0 . \\
2\end{array}$ & $\begin{array}{l}9 \\
9 \\
. \\
6\end{array}$ & $\begin{array}{l}9 \\
9 \\
. \\
3\end{array}$ & $\begin{array}{l}9 \\
9 \\
. \\
8\end{array}$ & $\begin{array}{l}1 \\
0 \\
0 . \\
1\end{array}$ & $\begin{array}{l}9 \\
9 \\
. \\
9\end{array}$ & $\begin{array}{l}1 \\
0 \\
0 . \\
0\end{array}$ & $\begin{array}{l}1 \\
0 \\
0\end{array}$ \\
\hline
\end{tabular}

Post Compression Parameters Of $10 \mathrm{Mg}$ Tablets

Table 08: Physical parameters of $10 \mathrm{mg}$ tablets

\begin{tabular}{|c|c|c|c|c|c|c|}
\hline $\begin{array}{c}\text { For- } \\
\text { mula- } \\
\text { tion } \\
\text { Code }\end{array}$ & $\begin{array}{c}\text { Av- } \\
\text { er- } \\
\text { age } \\
\text { wei } \\
\text { ght } \\
\text { (mg } \\
\text { ) }\end{array}$ & $\begin{array}{c}\text { Thic } \\
\text { knes } \\
\text { s } \\
\text { (mm } \\
\text { ) }\end{array}$ & $\begin{array}{l}\text { Har } \\
\text { dne } \\
\text { ss } \\
\text { (kp) }\end{array}$ & $\begin{array}{c}\text { Per- } \\
\text { cent- } \\
\text { age } \\
\text { Fri- } \\
\text { abil- } \\
\text { ity } \\
(\%)\end{array}$ & $\begin{array}{c}\text { Disin- } \\
\text { tegra- } \\
\text { tion } \\
\text { Time } \\
\text { (sec) }\end{array}$ & $\begin{array}{c}\text { Wet } \\
\text { ting } \\
\text { tim } \\
\text { e(se } \\
\text { c) }\end{array}$ \\
\hline F1 & $\begin{array}{c}199 . \\
23\end{array}$ & 2.97 & 1.12 & 1.29 & 22 & 14 \\
\hline F2 & $\begin{array}{c}200 . \\
01\end{array}$ & 3.01 & 2.08 & 0.21 & 21 & 13 \\
\hline F3 & $\begin{array}{c}200 . \\
03\end{array}$ & 2.89 & 1.77 & 0.36 & 12 & 9 \\
\hline $\mathrm{F} 4$ & $\begin{array}{c}199 . \\
89\end{array}$ & 2.94 & 1.75 & 0.38 & 11 & 7 \\
\hline F5 & $\begin{array}{c}200 . \\
01\end{array}$ & 3.01 & 1.78 & 0.43 & 15 & 12 \\
\hline F6 & $\begin{array}{c}200 . \\
12\end{array}$ & 2.99 & 1.80 & 0.42 & 20 & 14 \\
\hline F7 & $\begin{array}{c}199 . \\
98\end{array}$ & 3.03 & 1.76 & 0.40 & 14 & 9 \\
\hline F8 & $\begin{array}{c}200 . \\
00\end{array}$ & 3.00 & 1.75 & 0.39 & 10 & 7 \\
\hline F9 & $\begin{array}{c}200 . \\
02\end{array}$ & 3.01 & 1.74 & 0.39 & 12 & 7 \\
\hline F10 & $\begin{array}{c}200 . \\
00\end{array}$ & 2.98 & 1.79 & 0.35 & 11 & 8 \\
\hline
\end{tabular}

Table 10: Cumulative $\%$ drug release of $10 \mathrm{mg}$ tablets

\begin{tabular}{|c|c|c|c|c|c|c|c|c|c|c|c|c|}
\hline $\begin{array}{l}\mathrm{Ti} \\
\text { me } \\
\text { (m } \\
\text { in) }\end{array}$ & $\begin{array}{l}\mathbf{M} \\
\mathbf{A} \\
\mathbf{X} \\
\mathbf{A L} \\
\mathbf{T}\end{array}$ & $\begin{array}{l}F \\
1\end{array}$ & $\begin{array}{l}F \\
2\end{array}$ & $\begin{array}{l}F \\
3\end{array}$ & $\begin{array}{l}F \\
4\end{array}$ & $\begin{array}{l}F \\
5\end{array}$ & $\begin{array}{l}F \\
6\end{array}$ & $\begin{array}{l}\text { F } \\
7\end{array}$ & $\begin{array}{l}F \\
8\end{array}$ & $\begin{array}{l}F \\
9\end{array}$ & $\begin{array}{l}F \\
1 \\
0\end{array}$ & $\begin{array}{c}F \\
1 \\
1\end{array}$ \\
\hline 0 & 2 & 2 & 2 & 2 & 2 & 2 & 2 & 2 & 2 & 2 & 2 & 2 \\
\hline 5 & $\begin{array}{l}0.9 \\
9\end{array}$ & $\begin{array}{l}1 \\
. \\
1 \\
0 \\
3\end{array}$ & $\begin{array}{l}1 \\
. \\
0 \\
2 \\
1\end{array}$ & $\begin{array}{l}1 \\
. \\
0 \\
0 \\
4\end{array}$ & $\begin{array}{l}0 \\
. \\
9 \\
9 \\
5\end{array}$ & $\begin{array}{l}1 \\
. \\
0 \\
0 \\
4\end{array}$ & $\begin{array}{l}1 \\
. \\
0 \\
2 \\
9\end{array}$ & $\begin{array}{l}1 \\
. \\
0 \\
1 \\
2\end{array}$ & $\begin{array}{l}1 \\
. \\
0 \\
0 \\
8\end{array}$ & $\begin{array}{l}1 \\
\cdot \\
0 \\
1 \\
2\end{array}$ & $\begin{array}{l}1 \\
. \\
0 \\
0 \\
4\end{array}$ & $\begin{array}{l}1 \\
\cdot \\
0 \\
0 \\
8\end{array}$ \\
\hline 10 & $\begin{array}{l}0.6 \\
12\end{array}$ & $\begin{array}{l}0 \\
. \\
7 \\
4 \\
0\end{array}$ & $\begin{array}{l}0 \\
. \\
5 \\
9\end{array}$ & $\begin{array}{l}0 \\
. \\
6 \\
1 \\
2\end{array}$ & $\begin{array}{l}0 \\
. \\
4 \\
3 \\
1\end{array}$ & $\begin{array}{l}0 \\
. \\
4 \\
9 \\
1\end{array}$ & $\begin{array}{l}0 \\
. \\
5 \\
9 \\
1\end{array}$ & $\begin{array}{l}0 \\
. \\
5 \\
0 \\
5\end{array}$ & $\begin{array}{l}0 \\
. \\
4 \\
1 \\
4\end{array}$ & $\begin{array}{l}0 \\
\cdot \\
4 \\
7 \\
7\end{array}$ & $\begin{array}{l}0 \\
\cdot \\
4 \\
4 \\
7\end{array}$ & $\begin{array}{l}0 \\
\cdot \\
3 \\
8 \\
0\end{array}$ \\
\hline 15 & -1 & 0 & $\begin{array}{l}- \\
0 \\
. \\
0 \\
9\end{array}$ & $\begin{array}{l}- \\
0 \\
. \\
5 \\
2\end{array}$ & - & $\begin{array}{l}- \\
0 \\
. \\
6 \\
9 \\
8\end{array}$ & $\begin{array}{l}- \\
0 \\
. \\
1 \\
5 \\
4\end{array}$ & $\begin{array}{l}- \\
1\end{array}$ & - & $\begin{array}{l}- \\
0 \\
. \\
5 \\
2 \\
2\end{array}$ & $\begin{array}{l}- \\
1\end{array}$ & - \\
\hline
\end{tabular}


Cumulative $\%$ drug release of $10 \mathrm{mg}$ tablets

Table 10" Cumulative\% drug release of $10 \mathrm{mg}$ tablets

\begin{tabular}{|c|c|c|c|c|c|c|c|c|c|c|c|c|}
\hline $\begin{array}{l}\mathrm{S} \\
\cdot \\
\mathrm{N} \\
\mathbf{0}\end{array}$ & $\begin{array}{c}\text { Ti } \\
\text { me( } \\
\text { Mi } \\
\text { n) }\end{array}$ & $\begin{array}{l}F \\
1\end{array}$ & $\begin{array}{l}F \\
2\end{array}$ & $\begin{array}{l}F \\
3\end{array}$ & $\begin{array}{l}F \\
4\end{array}$ & $\begin{array}{l}F \\
5\end{array}$ & $\begin{array}{l}F \\
6\end{array}$ & $\begin{array}{l}F \\
7\end{array}$ & $\begin{array}{l}F \\
8\end{array}$ & $\begin{array}{l}F \\
9\end{array}$ & $\begin{array}{l}\mathbf{F} \\
\mathbf{1} \\
\mathbf{0}\end{array}$ & $\begin{array}{l}\mathbf{F} \\
\mathbf{1} \\
\mathbf{1}\end{array}$ \\
\hline 1 & 0 & 0 & 0 & 0 & 0 & 0 & 0 & 0 & 0 & 0 & 0 & 0 \\
\hline 2 & 5 & $\begin{array}{l}8 \\
8 \\
. \\
9\end{array}$ & $\begin{array}{l}8 \\
9 \\
. \\
5\end{array}$ & $\begin{array}{l}8 \\
9 \\
. \\
9\end{array}$ & $\begin{array}{l}9 \\
0 . \\
1\end{array}$ & $\begin{array}{l}8 \\
9 \\
. \\
9\end{array}$ & $\begin{array}{l}8 \\
9 \\
. \\
3\end{array}$ & $\begin{array}{l}8 \\
9 \\
. \\
7\end{array}$ & $\begin{array}{l}9 \\
0 . \\
0\end{array}$ & $\begin{array}{l}8 \\
9 \\
. \\
6\end{array}$ & $\begin{array}{l}8 \\
9 . \\
9\end{array}$ & $\begin{array}{l}8 \\
9\end{array}$ \\
\hline 3 & 10 & $\begin{array}{l}9 \\
5 \\
. \\
5\end{array}$ & $\begin{array}{l}9 \\
6 \\
. \\
1\end{array}$ & $\begin{array}{l}9 \\
6 \\
. \\
0\end{array}$ & $\begin{array}{l}9 \\
7 . \\
3\end{array}$ & $\begin{array}{l}9 \\
6 \\
9 \\
9\end{array}$ & $\begin{array}{l}9 \\
6 \\
. \\
1\end{array}$ & $\begin{array}{l}9 \\
6 \\
. \\
8\end{array}$ & $\begin{array}{l}9 \\
7 . \\
4\end{array}$ & $\begin{array}{l}9 \\
7 \\
. \\
0\end{array}$ & $\begin{array}{l}9 \\
7 . \\
2\end{array}$ & $\begin{array}{l}9 \\
7\end{array}$ \\
\hline 4 & 15 & $\begin{array}{l}9 \\
9 \\
. \\
0\end{array}$ & $\begin{array}{l}9 \\
9 \\
. \\
2\end{array}$ & $\begin{array}{l}9 \\
9 \\
. \\
6\end{array}$ & $\begin{array}{l}1 \\
0 \\
0 . \\
1\end{array}$ & $\begin{array}{l}9 \\
9 \\
. \\
8\end{array}$ & $\begin{array}{l}9 \\
9 \\
. \\
3\end{array}$ & $\begin{array}{l}9 \\
9 \\
. \\
9\end{array}$ & $\begin{array}{l}1 \\
0 \\
0 . \\
2\end{array}$ & $\begin{array}{l}9 \\
9 \\
. \\
9\end{array}$ & $\begin{array}{l}1 \\
0 \\
0 . \\
0\end{array}$ & 1 \\
\hline
\end{tabular}

\section{Log \% drug remained of $5 \mathrm{mg}$ tablet}

Table 11: $\log \%$ drug remained of $5 \mathrm{mg}$ tablets

\begin{tabular}{|c|c|c|c|c|c|c|c|c|c|c|c|c|}
\hline $\begin{array}{l}\mathrm{S} \\
\cdot \\
\mathrm{N} \\
\mathrm{o}\end{array}$ & $\begin{array}{l}\text { Tim } \\
\text { e(M } \\
\text { in) }\end{array}$ & $\begin{array}{l}F \\
1\end{array}$ & $\begin{array}{l}F \\
2\end{array}$ & $\begin{array}{l}F \\
3\end{array}$ & $\begin{array}{l}F \\
4\end{array}$ & $\begin{array}{l}F \\
5\end{array}$ & $\begin{array}{l}F \\
6\end{array}$ & $\begin{array}{l}F \\
7\end{array}$ & $\begin{array}{l}F \\
8\end{array}$ & $\begin{array}{l}F \\
9\end{array}$ & $\begin{array}{l}\text { F } \\
1 \\
0\end{array}$ & $\begin{array}{l}F \\
1 \\
1\end{array}$ \\
\hline 1 & 0 & 0 & 0 & 0 & 0 & 0 & 0 & 0 & 0 & 0 & 0 & 0 \\
\hline 2 & 5 & $\begin{array}{l}8 \\
8 \\
. \\
9\end{array}$ & $\begin{array}{l}8 \\
9 \\
. \\
5\end{array}$ & $\begin{array}{l}8 \\
9 \\
. \\
9\end{array}$ & $\begin{array}{l}9 \\
0 . \\
1\end{array}$ & $\begin{array}{l}8 \\
9 \\
. \\
9\end{array}$ & $\begin{array}{l}8 \\
9 \\
. \\
3\end{array}$ & $\begin{array}{l}8 \\
9 \\
. \\
7\end{array}$ & $\begin{array}{l}9 \\
0 . \\
0\end{array}$ & $\begin{array}{l}8 \\
9 \\
. \\
6\end{array}$ & $\begin{array}{l}8 \\
9 . \\
9\end{array}$ & $\begin{array}{l}8 \\
9\end{array}$ \\
\hline 3 & 10 & $\begin{array}{l}9 \\
5 \\
. \\
5\end{array}$ & $\begin{array}{l}9 \\
6 \\
. \\
1\end{array}$ & $\begin{array}{l}9 \\
6 \\
. \\
0\end{array}$ & $\begin{array}{l}9 \\
7 . \\
3\end{array}$ & $\begin{array}{l}9 \\
6 \\
. \\
9\end{array}$ & $\begin{array}{l}9 \\
6 \\
. \\
1\end{array}$ & $\begin{array}{l}9 \\
6 \\
. \\
8\end{array}$ & $\begin{array}{l}9 \\
7 . \\
4\end{array}$ & $\begin{array}{l}9 \\
7 \\
. \\
0\end{array}$ & $\begin{array}{l}9 \\
7 . \\
2\end{array}$ & $\begin{array}{l}9 \\
7\end{array}$ \\
\hline 4 & 15 & $\begin{array}{l}9 \\
9 \\
. \\
0\end{array}$ & $\begin{array}{l}9 \\
9 \\
. \\
2\end{array}$ & $\begin{array}{l}9 \\
9 \\
. \\
6\end{array}$ & $\begin{array}{l}1 \\
0 \\
0 . \\
1\end{array}$ & $\begin{array}{l}9 \\
9 \\
. \\
8\end{array}$ & $\begin{array}{l}9 \\
9 \\
. \\
3\end{array}$ & $\begin{array}{l}9 \\
9 \\
. \\
9\end{array}$ & $\begin{array}{l}1 \\
0 \\
0 . \\
2\end{array}$ & $\begin{array}{l}9 \\
9 \\
. \\
9\end{array}$ & $\begin{array}{l}1 \\
0 \\
0 . \\
0\end{array}$ & $\begin{array}{l}1 \\
0 \\
0\end{array}$ \\
\hline
\end{tabular}

Log \% drug remained of $10 \mathrm{mg}$ tablet

Table: $12 \log \%$ drug remained of $10 \mathrm{mg}$ tablets

\begin{tabular}{|c|c|c|c|c|c|c|c|c|c|c|c|c|}
\hline $\begin{array}{l}\mathrm{Ti} \\
\text { me } \\
\text { (M } \\
\text { in) }\end{array}$ & $\begin{array}{c}\mathbf{M} \\
\mathbf{A} \\
\mathbf{X} \\
\mathbf{A L} \\
\mathbf{T}\end{array}$ & $\begin{array}{l}F \\
1\end{array}$ & $\begin{array}{l}F \\
2\end{array}$ & $\begin{array}{l}F \\
3\end{array}$ & $\begin{array}{l}F \\
4\end{array}$ & $\begin{array}{l}F \\
5\end{array}$ & $\begin{array}{l}F \\
6\end{array}$ & $\begin{array}{l}\text { F } \\
7\end{array}$ & $\begin{array}{l}F \\
8\end{array}$ & $\begin{array}{l}F \\
9\end{array}$ & $\begin{array}{l}F \\
1 \\
0\end{array}$ & $\begin{array}{l}F \\
1 \\
1\end{array}$ \\
\hline 0 & 2 & 2 & 2 & 2 & 2 & 2 & 2 & 2 & 2 & 2 & 2 & 2 \\
\hline 5 & $\begin{array}{l}0.9 \\
95\end{array}$ & $\begin{array}{l}1 \\
. \\
1 \\
1\end{array}$ & $\begin{array}{l}1 \\
. \\
0 \\
2\end{array}$ & $\begin{array}{l}0 \\
. \\
9 \\
9 \\
5\end{array}$ & $\begin{array}{l}1 \\
. \\
0 \\
0 \\
8\end{array}$ & $\begin{array}{l}1 \\
. \\
0 \\
3 \\
3\end{array}$ & $\begin{array}{l}1 \\
. \\
0 \\
4 \\
5\end{array}$ & $\begin{array}{l}1 \\
. \\
0 \\
2 \\
1\end{array}$ & $\begin{array}{l}1 \\
. \\
0 \\
1 \\
2\end{array}$ & $\begin{array}{l}1 \\
. \\
0 \\
1 \\
7\end{array}$ & $\begin{array}{l}0 \\
0 \\
8\end{array}$ & 1 \\
\hline 10 & $\begin{array}{l}0.6 \\
33\end{array}$ & $\begin{array}{l}0 \\
. \\
7 \\
6\end{array}$ & $\begin{array}{l}0 \\
. \\
6 \\
1\end{array}$ & $\begin{array}{l}0 \\
. \\
6 \\
0 \\
2\end{array}$ & $\begin{array}{l}0 \\
. \\
4 \\
6 \\
2\end{array}$ & $\begin{array}{l}0 \\
. \\
5 \\
0 \\
5\end{array}$ & $\begin{array}{l}0 \\
5 \\
5 \\
6 \\
8\end{array}$ & $\begin{array}{l}0 \\
. \\
5 \\
0 \\
5\end{array}$ & $\begin{array}{l}0 \\
. \\
4 \\
3 \\
1\end{array}$ & $\begin{array}{l}0 \\
. \\
4 \\
6 \\
2\end{array}$ & $\begin{array}{l}4 \\
4 \\
7\end{array}$ & $\begin{array}{l}3 \\
9 \\
7\end{array}$ \\
\hline 15 & $\begin{array}{c}- \\
0.5 \\
2\end{array}$ & $\begin{array}{l}0 \\
7 \\
9\end{array}$ & $\begin{array}{l}- \\
0 \\
. \\
0 \\
4\end{array}$ & $\begin{array}{l}- \\
0 \\
. \\
2 \\
2 \\
1\end{array}$ & - & $\begin{array}{l}- \\
0 \\
. \\
3 \\
9 \\
7\end{array}$ & $\begin{array}{l}- \\
0 \\
\prime \\
1 \\
5 \\
4\end{array}$ & $\begin{array}{l}- \\
0 \\
. \\
6 \\
9\end{array}$ & - & $\begin{array}{l}- \\
1\end{array}$ & - & - \\
\hline
\end{tabular}


Results Of Stability Data For 5 Mg Tablets

Table 13: Stability study data for $5 \mathrm{mg}$ tablets

\begin{tabular}{|c|c|c|c|c|}
\hline \multirow{3}{*}{ Parameters Tested } & \multicolumn{4}{|c|}{ Storage Conditions } \\
\hline & \multirow{2}{*}{ INITIAL } & \multicolumn{3}{|c|}{$40^{\circ} \mathrm{C} \pm 2^{\circ} \mathrm{C} / 75 \% \pm 5 \% \mathrm{RH}$} \\
\hline & & $1^{\text {st }}$ Month & $2^{\text {nd }}$ Month & $3^{\text {rd }}$ Month \\
\hline Description & $\begin{array}{l}\text { White round tablets embossed with } 5 \text { on } \\
\text { one side and plain on the other side. }\end{array}$ & No change & No change & No change \\
\hline $\begin{array}{l}\text { Average weight } \\
\text { (mg) }\end{array}$ & 100.56 & 100.54 & 100.56 & 100.59 \\
\hline $\begin{array}{l}\text { Thickness } \\
\text { (mm) }\end{array}$ & 2.81 & 2.81 & 2.81 & 2.82 \\
\hline Hardness (kp) & 1.74 & 1.74 & 1.74 & 1.75 \\
\hline$\%$ Friability & 0.38 & 0.39 & 0.38 & 0.38 \\
\hline $\begin{array}{l}\text { Disintegration } \\
\text { time (sec) }\end{array}$ & 9 & 9 & 9 & 10 \\
\hline Water content (\%) & 1.253 & 1.252 & 1.249 & 1.250 \\
\hline
\end{tabular}

Results Of Stability Data For 10 Mg Tablets

Table 14: Stability study data for $10 \mathrm{mg}$ tablets

\begin{tabular}{|c|c|c|c|c|}
\hline \multirow{3}{*}{ Parameters Tested } & \multicolumn{4}{|c|}{ Storage Conditions } \\
\hline & \multirow{2}{*}{ INITIAL } & \multicolumn{3}{|c|}{$40^{\circ} \mathrm{C} \pm 2^{\circ} \mathrm{C} / 75 \% \pm 5 \% \mathrm{RH}$} \\
\hline & & $1^{\text {st }}$ Month & $2^{\text {nd }}$ Month & $3^{\text {rd }}$ Month \\
\hline Description & $\begin{array}{l}\text { White round tablets embossed with } 10 \text { on } \\
\text { one side and plain on the other side. }\end{array}$ & No change & No change & No change \\
\hline $\begin{array}{l}\text { Average weight } \\
\text { (mg) }\end{array}$ & 200 & 201 & 201 & 202 \\
\hline $\begin{array}{l}\text { Thickness } \\
\text { (mm) }\end{array}$ & 2.95 & 2.95 & 2.95 & 2.96 \\
\hline Hardness (kp) & 1.74 & 1.74 & 1.73 & 1.74 \\
\hline$\%$ Friability & 0.34 & 0.34 & 0.35 & 0.35 \\
\hline $\begin{array}{l}\text { Disintegration time } \\
\text { (sec) }\end{array}$ & 10 & 11 & 10 & 10 \\
\hline Water content $(\%)$ & 1.253 & 1.252 & 1.249 & 1.250 \\
\hline
\end{tabular}

\section{Summary and Conclusion}

The purpose of the present study was to develop and characterize a generic product of RizatriptanOral disintegrating tablets of strength $5 \mathrm{mg}$ and $10 \mathrm{mg}$; comparable to the brand product MAXALT-MLT(Merck. co. INC, USA).

To accomplish the objective, API characterization and reference product evaluation was carried out. The drug and excipients were subjected to preformulation studies which encompasses the "Drug-Excipient" compatibility. Direct compression technique was choosen to develop a finished pharmaceutical product of the envisaged form. Various formulation trials (F1-F11) were taken. In these trials, Drug: Excipient ratio was varied and the effect of Diluent, Superdisintegrant and lubricant on the performance of both blend as well as tablets was studied. Based on the results obtained it was concluded that the formulation F11, the reproducibility batch of F4 was finalized as the optimized formula. The final trial F11 was reproduced from formulation F4 to check various tablet parameters like Thickness (2.8-3.2)mm; Hardness (1.5-1.80)kp; Percentage Friability $(<1 \%)$ and Disintegra- 
tion time (<30 seconds), which were within the specified limits. The Dissolution and Assay results of F4 and F11 were good when compared with the reference product. Moisture uptake studies for the final batch F11 were performed at 43,64 and $75 \% \mathrm{RH}$ and there was a slight moisture uptake observed in tablets at 75\% RH. The reproducibility batch F11 was loaded for long term and accelerated stability studies at $25 \pm 2 \mathrm{C} / 60 \pm 5 \% \mathrm{RH}$ and $40 \pm 2 \mathrm{C} / 75 \pm 5 \% \mathrm{RH}$ respectively.. The results of stability data for $1^{\text {st }}, 2^{\text {nd }}$ month and 3rd months $(40 \pm 2 C / 75 \pm 5 \%$ $\mathrm{RH})$ were found to be good.

When subjected to accelerated stability studies the tablets were found to be stable. Thus, the work resulted in the development of a ODT of Rizatriptan comparable to inventors product.

\section{References}

1. Rangasamy Manivannan. Oral disintegrating tablets: A future Compaction Publication. International Journal of Pharmaceutical Research and Development 2009; 1: 1-10.

2. Mishra DN, Bindal M, Singh SK. Rapidly disintegrating oral tablet of valdecoxib. Indian drug. 2004; 41: 554.

3. Kaushik D, Dureja H, Saini TR. Mouth dissolving tablets: A review. Indian Drugs. 2004; 41: 503-508.

4. Ansel HC, Popovich NG, Allen LV. Pharmaceutical dosage forms and drug delivery system. B.I. Waverly Pvt. Ltd, New Delhi. 1995; 6:99-154.

5. Goodman, Gilman's. The Pharmacological Basis of Therapeutics. 11th ed. New York: McGraw Hill, 2006, pp 305-9.

6. Sanford B, Eds in pharmaceutical statistics, Practical and clinical applications, 2nd Edn, Marcel Dekker. NY, 1990, pp 303-337.

7. Wade A, and waul, P.J, Eds, in hand book of pharmaceutical excipients, ed 2, American pharmaceutical association, Washington, pharmaceutical press, London, 1994.

8. Banker G.S, Anderson N. R. In: Lachman L, Lieberman H.A. and Kanig J.L. The Theory and Practice of Industrial Pharmacy. 3rd ed, Mumbai, Varghese Publishing House, 1987, pp 293399.

9. Sreenivas S.A, Gadad A.P, Patil M.B. Formulation and evaluation of ondasetron hydrochloride directly compressed mouth disintegrating tablets. Indian Drugs, 2006: 43: 35-37.
10. Kundu S, Sahoo P.K. Recent trends in the developments of orally disintegrating technology. Pharma Times, 2008; 40: 11-15.

11. Gohel MC, Bansal G, Bhatt N. Formulation and evaluation of orodispersible taste masked tablets of famotidine. Pharma Biol World 2005; 3: 75-80.

12. United State Phamacopoeia . Convention. NF Asian edition, 2004, pp 74-75.

13. Marabathuni VJ, Dinesh P, Ravikumar R, Yamini P, Kiran PS, Hussain SP, Rao CM. Chitosan based sustained release mucoadhesive buccal patches containing amlodipine besylate (AMB). Asian J Res Pharm Sci. 2017 Jun 28;7:97104.

14. Marabathuni VJ, Bhavani M, Lavanya M, Padmaja K, Madhavi N, Babu P, Rao CM. Formulation and evaluation of mouth dissolving Tablets of carbamazepine. Asian Journal of Pharmacy and Technology. 2017;7(3):137-43. 